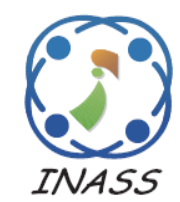

http://www.inass.org/

\title{
Performance of Max-Min User Relay Cooperative NOMA Systems with Imperfect CSI
}

\author{
Nam-Soo Kim ${ }^{1 *}$ \\ ${ }^{1}$ Department of Electronic Engineering, Cheongju University, Republic of Korea \\ * Corresponding author's Email: nskim@cju.ac.kr
}

\begin{abstract}
In this paper, we derive the effect of channel errors on the performance of user relaying cooperative NOMA system in closed-form. The max-min selection strategy is adapted to the selection of a relay among near users (NUs) and selection combining (SC) is applied to a far user (FU) for the performance improvements. As the previous studies found, the max-min relay selection without channel errors is effective to improve the outage performance. However, we demonstrate that the outage performance is highly sensitive to the channel error. It is also found that the outage probability of the relay user is more susceptible to the channel errors. As the channel correlation coefficient approaches to 0 , the performance gains are banishing and the outage probability with the max-min relay selection becomes identical to that with the random relay selection. NOMA system has an optimal power allocation coefficient to minimize the outage performance, but as the correlation coefficient decrease, the effect of power allocation to the performance becomes lessen. The provided numerical results confirm that the derived expressions match well with Monte Carlo simulations.
\end{abstract}

Keywords: NOMA, Cooperative NOMA, Max-min, Imperfect CSI, User relay.

\section{Introduction}

Next-generation cellular mobile systems demand more traffic capacity and higher data rates within the limited bandwidth. Recently introduced nonorthogonal multiple access (NOMA) has been focused for the promising candidate for the nextgeneration cellular networks. In NOMA, multiple users share both time and frequency resources with different power allocations. These lead to the higher spectral efficiency than the conventional orthogonal multiple access (OMA) [1-2].

Usually, a far user (FU) at the cell edge receives weak signal from a base station compared to the near users (NUs) in general cellular environments. This makes the more power allocation to $\mathrm{FU}$ to compensate the signal strength caused by the path loss and fading than NU in NOMA systems. Recently a cooperative communication with a relay is introduced in NOMA system to improve the performance degradation using a relay and/or space diversity against the fading in wireless channel.
There are two kinds of relaying method which are the dedicated relaying [3-4], and the user relaying [5-6]. Firstly, the dedicated relaying is the conventional relaying method which receives signals from a base station and transmits to users by amplify-and-forward or decode-and-forward relaying. Secondly, the user relaying utilizes an NU in NOMA instead of a standalone dedicated relay. In the process of the successive interference cancellation (SIC) which removes interferences from other users in NOMA system, the decoded information of FU is obtained and it can be used for the relaying [5]. In the case of a temporary network, i.e., an Ad-Hoc network, the user relaying is more preferable than the permanent dedicated relaying for simple and temporary network configuration.

Furthermore, we can apply the relay selection which improves the performance of a system when multiple candidate relays are exist. In [4, 7], the performance improvements in a cooperative NOMA system with relay selection under the assumption of perfect channel state information (CSI) have been 
analyzed. However, this assumption is not realistic in mobile fading environments. It is important to notice the time differences between the moments of the relay selection and the actual information transmission can cause channel errors in wireless fading channels. Consequently, these channel errors are seriously diminishing the performance gain obtained from the relay selection. Recently published studies of [8-10] consider the degradation incurred from the channel errors in NOMA system, but didn't expand to a cooperative system. In [9] and [10], the approximation and upper bound of the outage probability are derived, respectively. More recently, [11] discussed a cooperative NOMA with imperfect CSI, however, it considered the case of the dedicated relaying and did not include the relay selection.

Recently, we published user relay cooperative NOMA systems [12]. However, it assumed perfect channel state information, which does not include channel errors. Therefore, we consider a user relay cooperative NOMA system with channel errors. The max-min relay selection strategy is applied for the selection of a relay user (RU) among NUs in NOMA system. Also, the selection combining (SC) is adapted to FU for space diversity gain. We derive the outage probability of RU and FU in closed-form, respectively, since the information for RU and FU is different in NOMA systems. We showed the channel errors cause the sensitive degradation to the outage probability of RU and FU. To demonstrate the accuracy of the developed analytical results, we showed the simulation results perfectly match the analytical results.

The remainder of this paper is organized as follows. In section 2, the system model, channel errors, and relay selection strategy are described. Section 3 derives the outage probability of RU, FU, and the end-to-end performance in closed-form. The numerical results are presented and Monte Carlo simulations are applied to verify the accuracy of the derived analysis in section 4. Finally, section 5 concludes the paper.

\section{System model}

We consider a downlink NOMA system which consists of a base station as a source ( $S$ ), $N$ Relays $\left(R_{i}, i=1,2, \ldots, N\right)$, and $\mathrm{FU}$ as shown in Fig.1. In this paper, we consider the user relaying, therefore $N$ relays which are the NUs in the NOMA system are the candidate relays. We assume NUs are clustered relatively close together, hence the distances between $S$ and NUs and between NUs and FU are identical, respectively [3].

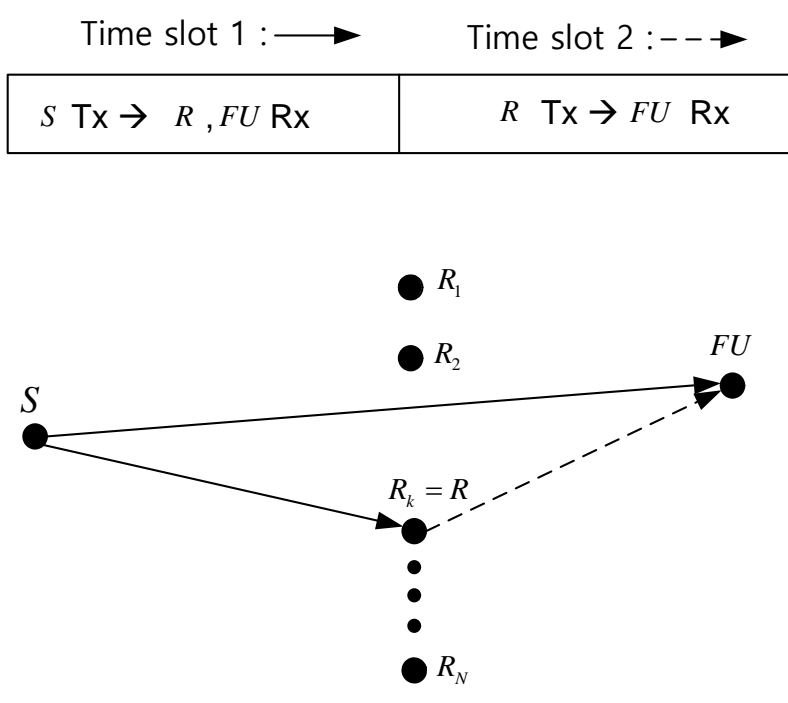

Figure. 1 System model

As mentioned in section 1 , the selected relay removes the interferences from FU with the SIC process. The decoded information for FU during the process can be utilized for relaying. In Fig.1, the selected relay of $R_{k}$, which is the best relay to maximize the performance of $\mathrm{FU}$, forwards the decoded information to FU. The max-min selection strategy is applied to select the best relay among NUs $[13,14]$. We assume the best relay is selected prior to information transmission for multiplexing $R_{k}$ and FU with different power levels in NOMA scheme.

The rapidly changing channel causes channel estimation errors. There are many factors to cause the channel estimation errors, feedback delay, fading and quantization errors. Statistically, the actual channel and the estimated channel can be represented by [1516]

$$
h=\rho \hat{h}+\sqrt{1-\rho^{2}} \varepsilon
$$

where $h$ denotes actual channel coefficient and assumed as a complex Gaussian random variable, $h \sim C N(0,1)$. And the estimated channel coefficient is denoted by $\hat{h} \sim C N(0,1) \quad . \quad \rho$ is the correlation coefficient between the actual and the estimated channel coefficients. $\varepsilon=C N(0,1)$ is independent of $\hat{h}$. All channels are assumed independent and identically distributed (i.i.d) Rayleigh block-fading channels; the channel response remains constant during one timeslot, but changes independently from one timeslot to another. We denote the actual channel coefficient and its estimated channel coefficient between node $\mathrm{A}$ and $\mathrm{B}$ as $h_{A B}$ and $\hat{h}_{A B}$, respectively. 
The max-min relay selection strategy which is known as a near optimal selection method to solve the bottleneck phenomenon can be described as follows; a central controller which knows every CSIs through pilot channels selects the less channel gain link of $S-R_{i}$ or $R_{i}-F U$ link in $S-R_{i}-F U$ path. Next, select maximum channel gain path $S-R_{i}-F U$ among the selected $N$ links (i.e., $S-R_{i}$ or $R_{i}-F U$ link, $i=1,2, \ldots, N)$ [4], [14]. The index of the selected best relay at the relay selection stage can be written by

$$
k=\arg \operatorname{maxmin}_{i=1,2, \ldots, N}\left(\left|\hat{h}_{S R_{i}}\right|^{2},\left|\hat{h}_{R_{i} F}\right|^{2}\right)
$$

For convenience, denote the selected relay $R_{k}$ by $R$. As shown in Fig.1, it requires two-time slots for the information transmission.

Time slot 1: Source transmit phase

At the first timeslot, a base station $S$ transmits the multiplexed message to the selected relay $R$ and FU based on the NOMA scheme, which is written by

$$
s=\sqrt{P_{S}}\left(\sqrt{\alpha_{R}} x_{R}+\sqrt{\alpha_{F}} x_{F}\right)
$$

where $P_{S}$ is the transmit power of the base station. $\alpha_{R}$ and $\alpha_{F}$ are the power allocation coefficient for $R$ and FU, respectively, where $\alpha_{F}>\alpha_{R}$ with $\alpha_{F}+\alpha_{R}=1 . x_{R}$ and $x_{F}$ denote the bipolar message symbol for $R$ and FU, respectively, with $\left|x_{R}\right|^{2}=\left|x_{F}\right|^{2}=1$.

The received signal of $R$ from $S-R$ path can be written by

$$
y_{S R}=\sqrt{P_{S R}}\left(\sqrt{\alpha_{R}} x_{R}+\sqrt{\alpha_{F}} x_{F}\right) h_{S R}+n_{R}
$$

where $P_{S R}$ denotes average received power at $R$, which can be defined by $P_{S R}=P_{S} d_{S R}^{-\alpha}$ where $d_{S R}$ is the distances between $S$ and $R$, and $\alpha$ is the propagation loss constant. $n_{R}$ denotes the Gaussian noise of $R, n \sim C N\left(0, \mathrm{~N}_{0}\right)$.

The received signal-to-interference plus noise ratio (SINR) of $R$ to detect $x_{F}$ can be written by

$$
\gamma_{S R}^{x_{F}}=\frac{\alpha_{F}\left|h_{S R}\right|^{2}}{\alpha_{R}\left|h_{S R}\right|^{2}+1 / \rho_{S R}}
$$

where $\rho_{S R}$ is the received signal-to-noise ratio (SNR) of $R, \rho_{S R}=P_{S R} / N_{0}$. After decoding $x_{F}$, this component is subtracted from the received signal by SIC. Thus the received instantaneous SNR of $R$ to detect $x_{R}$ after SIC can be written by

$$
\gamma_{S R}^{x_{R}}=\alpha_{R} \rho_{S R}\left|h_{S R}\right|^{2}
$$

The received signal of FU from $S-F U$ path can be written by

$$
y_{S F}=\sqrt{P_{S F}}\left(\sqrt{\alpha_{R}} x_{R}+\sqrt{\alpha_{F}} x_{F}\right) h_{S F}+n_{F}
$$

where $P_{S F}$ denotes the average received power of FU. $n_{F}$ denotes the Gaussian noise of the far user, $n_{F} \sim C N\left(0, \mathrm{~N}_{0}\right)$. Here we assumed the noise power of $R$ and FU is identical.

The received SINR of FU from Eq. (7) can be given by

$$
\gamma_{S F}^{x_{F}}=\frac{\alpha_{F}\left|h_{S F}\right|^{2}}{\alpha_{R}\left|h_{S F}\right|^{2}+1 / \rho_{S F}}
$$

where $\rho_{S F}$ is the received SNR of FU from $S-F U$ path, $\rho_{S F}=P_{S F} / N_{0}$.

Time slot 2: Relaying and combining phase

At this time slot, the selected decode-and-forward relay $(R)$ transmits to FU. The received signal of FU from $R-F U$ path can be written by

$$
y_{R F}=\sqrt{\alpha_{F} P_{R F}} h_{R F} x_{F}+n_{F}
$$

where $P_{R F}$ denotes average received power at FU.

The received SNR is given by

$$
\gamma_{R F}^{x_{F}}=\alpha_{F} \rho_{R F}\left|h_{R F}\right|^{2}
$$

where $\rho_{R F}$ is the received SNR of FU from $R-F U$ path, $\rho_{R F}=P_{R F} / N_{0}$.

The received signals from both the indirect $(R-F U)$ path and the direct $(S-F U)$ path are combined by SC at FU. 


\section{Outage probability}

The information for $R$ is different from FU in NOMA, the outage probability of $R$ and FU is derived respectively. Also the system outage probability of $R$ and FU paired is derived.

\subsection{Outage probability of $R$}

The outage of the selected relay is happened in two cases. First, SINR of the far user information of $x_{D}$ bellows the predefined threshold, hence $x_{D}$ cannot be decoded. On the other hand, SNR of the selected relay after SIC bellows threshold, hence $x_{R}$ cannot be decoded. Therefore, the outage probability of the selected relay can be written by

$$
P_{O, R}=\operatorname{Pr}\left(\gamma_{S R}^{x_{F}}<\Gamma_{F}\right)+\operatorname{Pr}\left(\gamma_{S R}^{x_{F}} \geq \Gamma_{F}, \gamma_{S R}^{x_{R}}<\Gamma_{R}\right)
$$

where $\Gamma_{F}$ and $\Gamma_{R}, \Gamma_{F}=2^{2 R_{F}}-1$ and $\Gamma_{R}=2^{R_{R}}-1$, are the threshold SNR. $R_{F}$ and $R_{R}$ are the spectral efficiency of FU and $R$, respectively.

Replacing Eq. (5) into the first probability of Eq. (11), we can write

$$
\begin{aligned}
\operatorname{Pr}\left(\gamma_{S R}<\Gamma_{F}\right) & =\operatorname{Pr}\left(\frac{\alpha_{F}\left|h_{S R}\right|^{2}}{\alpha_{R}\left|h_{S R}\right|^{2}+1 / \rho_{S R}}<\Gamma_{F}\right) \\
& =\operatorname{Pr}\left(\left|h_{S R}\right|^{2}<\varsigma\right)
\end{aligned}
$$

where $s=\Gamma_{F}\left(\alpha_{F}-\Gamma_{F} \alpha_{R}\right) \rho_{S R}$, and $\Gamma_{F}<\alpha_{F} / \alpha_{R}$. And a part of the second probabilities,

$$
\begin{aligned}
\operatorname{Pr}\left(\gamma_{S R}^{x_{R}}<\Gamma_{R}\right) & =\operatorname{Pr}\left(\alpha_{R} \rho_{S R}\left|h_{S R}\right|^{2}<\Gamma_{R}\right) \\
& =\operatorname{Pr}\left(\left|h_{S R}\right|^{2}<\eta\right)
\end{aligned}
$$

where $\eta=\Gamma_{R} / \alpha_{R} \rho_{S R}$.

Consequently, the outage probability of $R$ from Eq. (12) and Eq. (13) can be written by

$$
\begin{aligned}
P_{O, R} & =\left\{\begin{array}{l}
\operatorname{Pr}\left(\left|h_{S R}\right|^{2}<\varsigma\right), \varsigma \geq \eta \\
\operatorname{Pr}\left(\left|h_{S R}\right|^{2}<\eta\right), \varsigma<\eta
\end{array}\right. \\
& =F_{H_{R}}\{\max (\varsigma, \eta)\}
\end{aligned}
$$

where $\left|h_{S R}\right|^{2}$ is replaced to $H_{R},\left|h_{S R}\right|^{2}=H_{R}$, for notational simplicity in the last equality. $F_{H_{R}}\{\max (\varsigma, \eta)\}$ denotes the cumulative distribution function(CDF) of $H_{R}$. We will derive the probability distribution function (pdf) of $f_{H_{R}}(\bullet)$, firstly. Then, the CDF of $F_{H_{R}}(\bullet)$ can be obtained from $f_{H_{R}}(\bullet)$ by taking integration.

On the other hand, the actual channel and the estimated channel is different as mentioned in Eq. (1). Therefore the pdf of $H_{R}$ can be obtained from the conditional pdf and written by

$$
f_{H_{R}}(z)=\int_{0}^{\infty} f_{H_{R} \mid \hat{H}_{R}}(z \mid x) f_{H_{R}}(x) d x
$$

where $f_{H_{R} \mid \hat{H}_{R}}(z \mid x)$ denotes the conditional pdf with channel errors, and given by [17-18]

$$
f_{H_{R} \mid \hat{H}_{R}}(z \mid x)=\frac{1}{1-\rho^{2}} e^{-\frac{z+\rho^{2} x}{1-\rho^{2}}} I_{o}\left(\frac{2 \rho \sqrt{z x}}{1-\rho^{2}}\right) \text {. }
$$

Also the pdf of $\hat{H}_{R}, f_{\hat{H}_{R}}(x)$, denotes the pdf of the selected max-min relay in Eq. (15). From Eq. (23) in [12] which can be obtained from Eq. (2) in [14], it is given by

$$
\begin{aligned}
f_{\hat{H}_{R}}(x)= & \sum_{i=1}^{N}\left(\begin{array}{l}
N \\
i
\end{array}\right)(-1)^{i-1} \frac{i}{1-2 i}\left(e^{-2 i x}-e^{-x}\right) \\
& +\sum_{i=1}^{N}\left(\begin{array}{l}
N \\
i
\end{array}\right)(-1)^{i-1} i e^{-2 i x}
\end{aligned}
$$

Replacing Eqs. (16) and (17) into Eq. (15), we can obtain

$$
\begin{aligned}
f_{H_{R}}(z)= & \frac{1}{1-\rho^{2}} \mathrm{e}^{-z\left(1-\rho^{2}\right)} \sum_{i=1}^{N}\left(\begin{array}{l}
N \\
i
\end{array}\right)(-1)^{-1} \frac{1}{1-2 i} \int_{0}^{\infty} \mathrm{e}^{-\left(\frac{\rho^{2}}{1-\rho^{2}}+2 i\right) x} I_{0}\left(\frac{2 \rho \sqrt{Z X}}{1-\rho^{2}}\right) d x \\
& -\frac{1}{1-\rho^{2}} \mathrm{e}^{-z\left(1-\rho^{2}\right)} \sum_{i=1}^{N}\left(\begin{array}{l}
N \\
i
\end{array}\right)(-1)^{i-1} \frac{1}{1-2 i} \int_{0}^{\infty} \mathrm{e}^{-\left(\frac{\rho^{2}}{1-\rho^{2}}+1\right) x} I_{0}\left(\frac{2 \rho \sqrt{Z X}}{1-\rho^{2}}\right) d x \\
& +\frac{1}{1-\rho^{2}} \mathrm{e}^{-z\left(1-\rho^{2}\right)} \sum_{i=1}^{N}\left(\begin{array}{l}
N \\
i
\end{array}\right)(-1)^{i-1} i \int_{0}^{\infty} \mathrm{e}^{-\left(\frac{\rho^{2}}{1-\rho^{2}}+2 i\right) x} I_{0}\left(\frac{2 \rho \sqrt{Z X}}{1-\rho^{2}}\right) d x
\end{aligned}
$$




$$
\begin{aligned}
\int_{0}^{\infty} e^{-\alpha x} I_{2 v}(2 \sqrt{\beta x}) d x & =\frac{1}{\sqrt{\alpha \beta} \Gamma(2 v+1)} e^{\frac{\beta}{2 \alpha}} \Gamma(v+1) \\
& \times M_{-1 / 2, v}\left(\frac{\beta}{\alpha}\right), \alpha>0, v>-1
\end{aligned}
$$

where

$$
M_{\lambda, \mu}(z)=z^{\mu+1 / 2} e^{-z / 2} \Phi\left(\mu-\lambda+\frac{1}{2}, 2 \mu+1 ; z\right)(20)
$$

and where $\Phi(\alpha, \alpha, z)=e^{z}$ [19, (9.215.1)]. Appling Eqs. (19) and (20) to Eq. (18), we can rearrange the pdf in closed-form

$$
\begin{aligned}
f_{H_{R}}(z)= & \sum_{i=1}^{N}\left(\begin{array}{l}
N \\
i
\end{array}\right)(-1)^{i-1} \frac{i}{1-2 i} \frac{1}{\rho^{2}+2\left(1-\rho^{2}\right) i} e^{-\frac{z}{1-\rho^{2}}\left\{1-\frac{\rho^{2}}{\rho^{2}+2\left(1-\rho^{2}\right)}\right\}} \\
& -\sum_{i=1}^{N}\left(\begin{array}{l}
N \\
i
\end{array}\right)(-1)^{i-1} \frac{i}{1-2 i} e^{-z} \\
& +\sum_{i=1}^{N}\left(\begin{array}{l}
N \\
i
\end{array}\right)(-1)^{i-1} \frac{i}{\rho^{2}+2\left(1-\rho^{2}\right) i} e^{-\frac{z}{1-\rho^{2}}\left\{1-\frac{\rho^{2}}{\rho^{2}+2\left(1-\rho^{2}\right) i}\right\}}
\end{aligned}
$$

By integrating Eq. (21), the CDF of $H_{R}$ can be written by

$$
\begin{aligned}
F_{H_{R}}(y)= & \int_{0}^{y} f_{H_{R}}(z) d z \\
& =\sum_{i=1}^{N}\left(\begin{array}{l}
N \\
i
\end{array}\right)(-1)^{i-1} \frac{i}{1-2 i} \frac{1}{\rho^{2}+2\left(1-\rho^{2}\right) i} \Phi_{a}(y) \\
& -\sum_{i=1}^{N}\left(\begin{array}{l}
N \\
i
\end{array}\right)(-1)^{i-1} \frac{i}{1-2 i}\left(1-e^{-y}\right) \\
& +\sum_{i=1}^{N}\left(\begin{array}{l}
N \\
i
\end{array}\right)(-1)^{i-1} \frac{i}{\rho^{2}+2\left(1-\rho^{2}\right) i} \Phi_{a}(y)
\end{aligned}
$$

where $\quad \Phi_{a}(y)=\frac{1}{a}\left(1-e^{-a y}\right)$

and

$$
a=\frac{1}{1-\rho^{2}}\left\{1-\frac{\rho^{2}}{\rho^{2}+2\left(1-\rho^{2}\right) i}\right\} .
$$

Therefore, $F_{H_{R}}\{\max (\varsigma, \eta)\}$ can be obtained from substituting $y$ by $\max (\varsigma, \eta)$ in Eq. (22). As a special case of $\rho=1$, which means error free, Eq. (22) becomes identical to [12, (23)].

\subsection{Outage probability of FU}

The received signals both from the indirect path $(R-F U$ path) and from the direct path $(S-F U$ path) are combined at FU with SC. Therefore, the outage probability of $\mathrm{FU}$ can be obtained by multiplying the outage probability of the indirect path and that of the direct path, and can be written by

$$
P_{o, F}=P_{o, \text { ind }} \times P_{o, \text { dir }}
$$

where $P_{o, \text { ind }}$ and $P_{o, \text { dir }}$ denote the outage probability of the indirect path and the direct path, respectively. The indirect path are composed of $S-R$ and $R-F U$ links. When one of the link fails, the outage happens. The outage probability of FU through indirect path can be written by

$$
\begin{aligned}
P_{o, \text { ind }} & =\operatorname{Pr}\left\{\min \left(\gamma_{S R}^{x_{F}}, \gamma_{R F}^{x_{F}}\right)<\Gamma_{F}\right\} \\
& =1-\operatorname{Pr}\left(\gamma_{S R}^{x_{F}} \geq \Gamma_{F}\right) \operatorname{Pr}\left(\gamma_{R F}^{x_{F}} \geq \Gamma_{F}\right) \\
& =1-\operatorname{Pr}\left(H_{R} \geq \varsigma\right) \operatorname{Pr}\left(H_{R F} \geq \lambda\right) \\
& =1-\left\{1-F_{H_{R}}(\varsigma)\right\}\left\{1-F_{H_{R F}}(\lambda)\right\}
\end{aligned}
$$

where $H_{R F}=\left|h_{R F}\right|^{2}$ and $\lambda=\Gamma_{F} / \rho_{R F} \alpha_{F}$. Similarly, the pdf of $H_{R F}$ can be obtained from Eq. (15) by replacing $H_{R F}$ and $\hat{H}_{R F}$ instead of $H_{R}$ and $\hat{H}_{R}$, respectively. We can write

$$
f_{H_{R F}}(z)=\int_{0}^{\infty} f_{H_{R F} \mid \hat{H}_{R F}}(z \mid x) f_{\hat{H}_{R F}}(x) d x .
$$

Also the $\mathrm{CDF}$ of $F_{H_{R F}}(\lambda)$ can be obtained by substituting $H_{R F}$ and $\lambda$ by $H_{R}$ and $y$ in Eq. (22), respectively.

On the other hand, the outage probability of FU through direct path can be derived similar to Eq. (12), and can be written by

$$
P_{o, \text { dir }}=\operatorname{Pr}\left(\gamma_{S F}^{x_{F}}<\Gamma_{F}\right)=\operatorname{Pr}\left(\left|h_{S F}\right|^{2}<\chi\right)=1-e^{-x}
$$

where $\chi=\Gamma_{F} / \rho_{S F}\left(\alpha_{F}-\alpha_{R} \Gamma_{F}\right), \quad \rho_{S F}=P_{S F} / N_{0}$, and $P_{S F}=P_{S} d_{S F}^{-\alpha}$ which is the average received power from direct path. The last equality of Eq. (26) assumed Rayleigh fading.

Finally, the outage probability of FU with SC can be obtained by replacing Eqs. (24) and (26) into Eq. (23). 


\subsection{Outage probability of NOMA system}

The outage of NOMA system happens either $R$ or FU is in outage. Hence the end-to-end outage probability of NOMA system can be defined by

$$
P_{o, s y s}=1-\left(1-P_{o, R}\right)\left(1-P_{o, F}\right) \text {. }
$$

By inserting Eqs. (14) and (23) into Eq. (27), we can written

$$
\begin{aligned}
P_{O, S Y S}= & 1-\left[1-F_{H_{R}}\{\max (\varsigma, \eta)\}\right] \\
& \times\left[1-\left\{F_{H_{R}}(\varsigma)+F_{H_{R F}}(\lambda)-F_{H_{R}}(\varsigma) F_{H_{R F}}(\lambda)\right)\left(1-e^{-\chi}\right)\right] .
\end{aligned}
$$

\section{Numerical examples}

Fig. 2 shows the outage probability of the selected relay user $R$, where the solid lines denote the analytical results and "o" indicates the Monte Carlo simulation results. The analytical results and the simulation results are perfectly matched.

In Fig. 2, the curb "Random" is also plotted for reference, where "Random" means that the relay is selected randomly among NUs. While "Maxmin" denotes the relay with the max-min selection strategy. Especially, the outage probability with $\rho=1$ displays the special case of no channel error, which coincides with our previous results in [12]. The performance improvements with the max-min relay selection decrease as the value of $\rho$ decreases. When $\rho=0$, the outage probability coincides with that of the random selection. However, we noticed that the performance improvement with the max-min relay selection is highly effective at low channel error, that means high correlation coefficient $\rho$.

The outage probability of FU versus average SNR shows in Fig. 3. In this figure, the curb "Direct" is also plotted for reference, where "Direct" means the outage probability of FU with the direct path $(S-F U$ path) only. Irrespective of relay selection method, max-min or random relay selection, we noticed that the performance of the cooperative system is better than that of the direct path only.

Similar to the results of Fig. 2, the performance with the max-min relay selection improves as $\rho$ approaches to 1 . On the contrary, the performance degrades as $\rho$ approaches to 0 , and finally identical to that of the random selection. It is noticed that the effect of the channel error to the performance of FU is less sensitive compared to that of RU, since the space diversity of SC compensates the degradation.

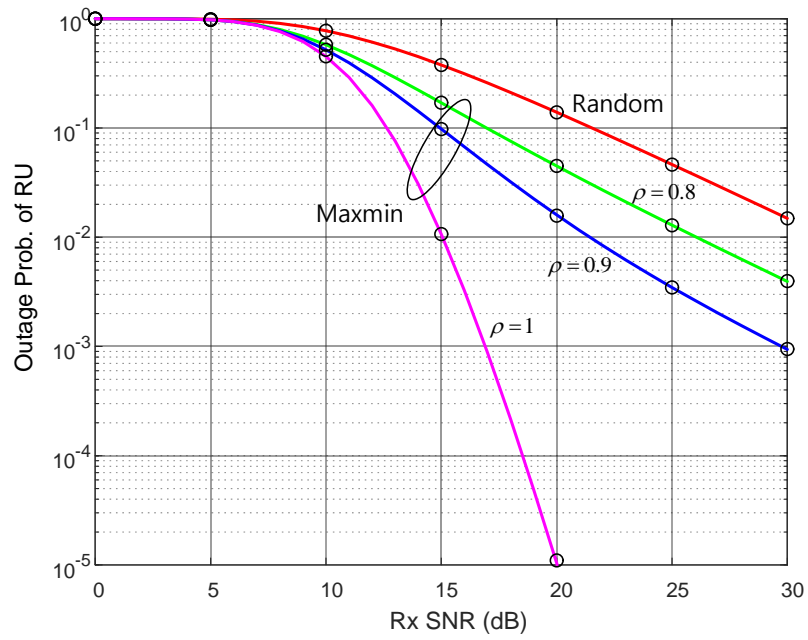

Figure. 2 Outage probability of Relay user $\left(R_{F}=R_{R}=1, N=8, \rho_{S R}=\rho_{R F}, \alpha_{F}=0.8\right)$

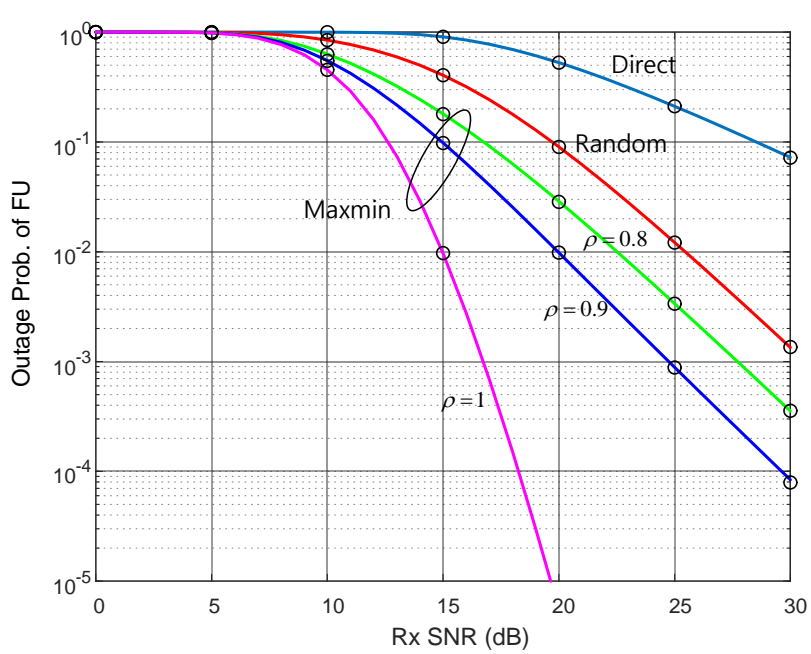

Figure. 3 Outage probability of Far user $\left(R_{F}=R_{R}=1\right.$, $\left.N=8, \rho_{S R}=\rho_{R F}, \rho_{S F}=0.2 \rho_{R F}, \alpha_{F}=0.8\right)$

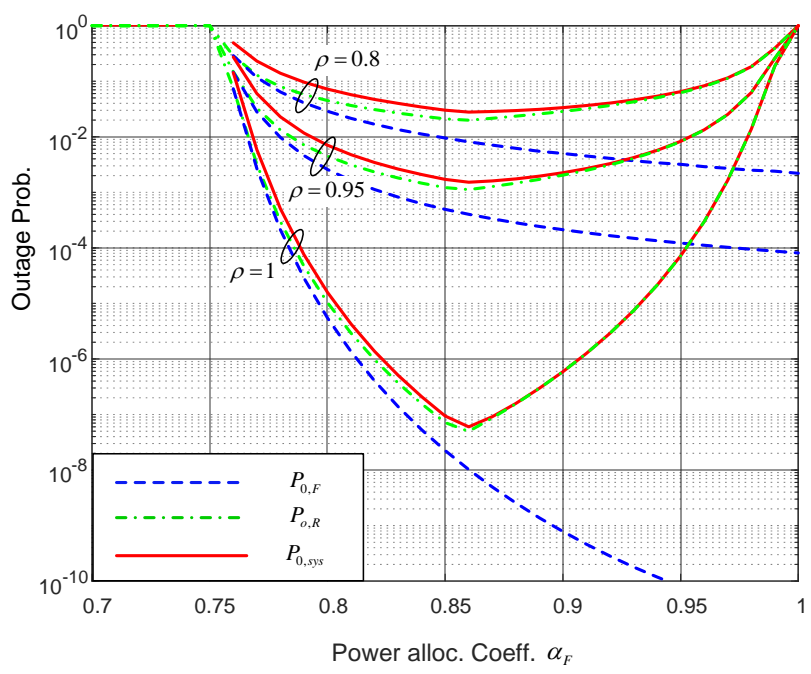

Figure. 4 Outage probability vs. power allocation for different correlation coefficients $\left(R_{F}=R_{R}=1, N=8\right.$,

$$
\left.\rho_{S R}=\rho_{R F}=20 d B, \rho_{S F}=0.2 \rho_{R F}\right)
$$


The effect of the power allocation to the outage probabilities are shown in Fig. 4. The increase of $\alpha_{F}$ means the power allocation to FU increases in Eq. (3), consequently the outage probability of FU decreases. However the total transmit power is limited to $\alpha_{F}+\alpha_{R}=1$, it is noticed that the optimal power allocation to minimize the outage probability of $R$ exists at the given condition as shown in Fig.4. Also we noticed that the system outage probability dominantly depends on the outage probability of relay $R$.

This Fig.4 shows the power allocation coefficient to minimize the outage probability under given conditions is approximately 0.86 irrespective of the channel correlation coefficient. As the channel correlation coefficient decrease, the effect of power allocation to the performance becomes lessen.

\section{Conclusions}

In this paper, we consider a user relay cooperative NOMA system with channel estimation errors. The channel estimation errors are modeled by a correlation coefficient between the actual channel and the estimated channel. The max-min relay selection strategy is applied for the relay selection among NUs and the selection combining is assumed for the space diversity to FU. The outage performances of RU and FU as a function of the correlation coefficient are derived analytically in closed-form, respectively. The derived performances are verified by Monte Carlo simulation.

As the previous studies found, the max-min relay selection without channel errors $(\rho=1)$ is effective to improve the outage performance compared to the random selection which is shown in Fig.2 and Fig.3. However, these figures demonstrate that the outage probabilities of RU and FU are affected by the channel correlation. In Fig.2 and Fig.3, to maintain the outage probability of $1 \times 10^{-3}$ with $\rho=0.9$, the relay user and the far user require more than $13 \mathrm{~dB}$ and $8 \mathrm{~dB}$ of SNR compared with $\rho=1$, respectively. It represents that the outage probability of the relay user is more susceptible to the channel errors. It is interpreted that the spatial diversity at far user which combine both signals from the direct and indirect paths mitigates the performance degradation caused by channel errors.

Moreover, the channel correlation coefficient approaches to 0 , the performance gains are banishing and the outage probability with the max-min relay selection becomes identical to that with the random relay selection. From this result, we can conclude that the effect of the channel errors to the outage performance is not negligible. The generally accepted performance gain from the max-min relay selection can be banished as the channel errors increasing.

NOMA system has an optimal power allocation coefficient to minimize the outage performance, but as the correlation coefficient decrease, the effect of power allocation to the performance becomes lessen. Further research will be focused on the performance of cooperative NOMA systems in general fading.

\section{References}

[1] L. Song, Y. Li, Z. Ding, and H. V. Poor, "Resource management in non-orthogonal multiple access networks for 5G and beyond", IEEE Network, Vol.31, No.4, pp.8-14, 2017.

[2] J. Zhao, Z. Ding, P. Fan, Z. Yang, and G. Karagiannidis, "Dual relay selection for cooperative NOMA with distributed space time coding", IEEE Access, Vol.6, pp.20440-20450, 2018.

[3] P. Xu, Z. Yang, Z. Ding, and Z. Zhang, "Optimal relay selection schemes for cooperative NOMA", IEEE Transactions on Vehicular Technology, to be appear in April 2018.

[4] Z. Ding, H. Dai, and H. V. Poor, "Relay selection for cooperative NOMA", IEEE Wireless Communications Letters, Vol.5, No.4, pp.416419, 2016.

[5] Z. Ding, M. Peng, and H. V. Poor, "Cooperative non-orthogonal multiple access in $5 \mathrm{G}$ systems", IEEE Communications Letters, Vol.19, No.8, pp.1462-1465, 2015.

[6] Y. Liu, Z. Ding, M. Elkashlan, and H. V. Poor, "Cooperative non-orthogonal multiple access with simultaneous wireless information and power transfer", IEEE Journal on Selected Areas in Communications, Vol.14, No.4, pp.938-953, 2016.

[7] S. Lee, D. B. da Costa, and T. Q. Duong, "Outage probability of non-orthogonal multiple access schemes with partial relay selection", In: Proc. of Personal, Indoor, and Mobile Communications, pp.1-6, 2016.

[8] F. Fang, H. Zhang, J. Cheng, and V. C. M. Leung, "Energy-efficient resource scheduling for NOMA systems with imperfect channel state information", In: Proc. of International Conference on Communications, pp.1-5, 2017.

[9] Z. Yang, Z. Ding, P. Fan, and G. K. Karagiannidis, "On the performance of non-orthogonal multiple access systems with partial channel information", IEEE Transactions on Communications, Vol.64, No.2, pp.654-667, 2016. 
[10] W. Cai, C. Chen, L. Bai, Y. Jin, and J. Choi, "User selection and power allocation schemes for downlink NOMA systems with imperfect CSI", In: Proc. of Vehicular Technology Conference, pp.1-5, 2016.

[11] D. Wan, M. Wen, F. Ji, Y. Liu, and Y. Huang, "Cooperative NOMA systems with partial channel state information over Nakagami-m fading channels", IEEE Transactions on Communications, Vol.66, No.3, pp.947-958, 2017.

[12] N.-S. Kim, "Utilization of max-min user relay in cooperative NOMA systems", Journal of Telecommunications, Electronic and Computer Engineering, Vol.10, No.1-4, pp.89-94, 2018.

[13] A. Bletsas, H. Shin, and M. Z. Win, "Cooperative communications with outageoptimal opportunistic relaying", IEEE Transactions on Wireless Communications, Vol.6, No.9, pp.3450-3460, 2007.

[14] K. Tourki, H.-C. Yang, and M.-S. Alouini, "Accurate outage analysis of incremental decodeand-forward opportunistic relaying", IEEE Transactions on Wireless Communications, Vol.10, No.4, pp.1021-2015, 2011.

[15] H. Sun and M. N.- Pour, "Decode-and-forward relay selection with imperfect CSI in cognitive network", In: Proc. of Military Communications Conf., pp.416-421, 2014.

[16] V. Sharma, K. Premkumar, and R. N. Swamy, "Exponential diversity achieving spatio-temporal power allocation scheme for fading channels," IEEE Transactions on Information Theory, Vol. 54, No.1, pp.188-208, 2008.

[17] J. L. Vicario and C. Anton-Haro, "Analytical assessment of multi-user vs. spatial diversity trade-offs with delayed channel state information", IEEE Communications Letters, Vol.10, No.8, pp.588-590, 2006.

[18] D. S. Michalopoulos, H. A. Suraweera, G. K. Karagiannidis, and R. Schober, "Amplify-andforward relay selection with outdated channel estimates", IEEE Transactions on Communications, Vol.60, No.5, pp.1278-1290, 2012.

[19] I. S. Gradshteyn and I. M. Ryzbik, Table of integrals, series, and products, $6^{\text {th }}$ ed., Academic Press, London, 2000. 